\title{
On the scaling and ageing behaviour of the alternating susceptibility in spin glasses and local scale-invariance $\ddagger$
}

\author{
Malte Henkel ${ }^{1}$ and Michel Pleimling ${ }^{2}$ \\ ${ }^{1}$ Laboratoire de Physique des Matériaux, $\S$ Université Henri Poincaré Nancy I, \\ B.P. 239, F - 54506 Vandœuvre lès Nancy Cedex, France \\ ${ }^{2}$ Institut für Theoretische Physik I, Universität Erlangen-Nürnberg, \\ Staudtstraße 7B3, D - 91058 Erlangen, Germany
}

\begin{abstract}
The frequency-dependent scaling of the dispersive and dissipative parts of the alternating susceptibility is studied for spin glasses at criticality. An extension of the usual $\omega t$-scaling is proposed. Simulational data from the three-dimensional Ising spin glass agree with this new scaling form and moreover reproduce well the scaling functions explicitly calculated for systems satisfying local scale-invariance. There is also a qualitative agreement with existing experimental data.
\end{abstract}

PACS numbers: 05.70.Ln, 75.50.Lk, 64.60.Ht, 11.25.Hf

Submitted to: J. Phys.: Condens. Matter

$\ddagger$ Dedicated to Lothar Schäfer on the occasion of his $60^{\text {th }}$ birthday.

$\S$ Laboratoire associé au CNRS UMR 7556 


\section{Introduction}

Understanding the complex behaviour of glass-forming systems cooled to below their glass transition temperature remains a challenge. At first sight, the problem might appear to be hopelessly difficult, since time-translation invariance is in general broken and the properties of observables may hence depend on the prehistory of the material under study (i.e. thermal, mechanical,...). On the other hand, an important discovery has been the observation of dynamical scaling, see [1], which occurs quite independently of whether the equilibrium state is critical or not. In recent years, it has been realized that many aspects of dynamical scaling are conveniently first studied in non-disordered, i.e. ferromagnetic systems. After a quench to or below their critical temperature $T_{c}$, these systems undergo an ageing behaviour which in many respects is quite similar to the one in glassy or kinetically constrained systems. The manifold problems which arise in the study of ageing in simple magnets or glasses are reviewed, e.g. in $[2,3,4,5,6,7,8,9]$.

For notational simplicity, we shall in what follows consider magnetic spin systems. Convenient tools for the study of ageing behaviour are the two-time autocorrelation and autoresponse functions

$$
\begin{aligned}
& C(t, s)=\langle\phi(t) \phi(s)\rangle \sim s^{-b} f_{C}(t / s) \\
& R(t, s)=\left.\frac{\delta\langle\phi(t)\rangle}{\delta h(s)}\right|_{h=0} \sim s^{-1-a} f_{R}(t / s)
\end{aligned}
$$

where $\phi(t)$ is the order parameter at time $t$ and $h(s)$ is the conjugate magnetic field at time $s$. The scaling behaviour is expected to apply in the so-called ageing regime where $t, s \gg t_{\text {micro }}$ and $t-s \gg t_{\text {micro, }}$, where $t_{\text {micro }}$ is a microscopic time scale. Furthermore, we tacitly assumed that the scaling derives from the time-dependence of a single characteristic length-scale $L(t)$ which measures the linear size of correlated clusters. In this paper, we shall always consider algebraic growth laws, viz. $L(t) \sim t^{1 / z}$, where $z$ is the dynamic exponent. Then the above forms define the nonequilibrium exponents $a$ and $b$ and the scaling functions $f_{C}(y)$ and $f_{R}(y)$. For large arguments $y \rightarrow \infty$, one generically expects

$$
f_{C}(y) \sim y^{-\lambda_{C} / z}, f_{R}(y) \sim y^{-\lambda_{R} / z}
$$

where $\lambda_{C}$ and $\lambda_{R}$, respectively, are known as autocorrelation $[10,11]$ and autoresponse exponents [12]. This description applies to many simple magnets quenched to a temperature $T \leq T_{c}$ as is well-known, see $[2,3,5] . \dagger$ On the other hand, for glasses quenched to below the glass-transition temperature, a slow cross-over between an algebraic growth law at short times towards a slower (logarithmic) growth at larger times appears to give a better description of the data, see [8] and references therein. Recently, evidence was found that for spin glasses quenched onto their critical temperature $T=T_{c}$ a simple scaling of the two-time observables compatible with an algebraic growth law applies [13].

$\dagger$ Exceptions occur for example in the $2 D \mathrm{XY}$ model with a fully disordered initial state. 
In recent years, from the study of ageing in simple magnets it has been proposed that global dynamical scaling might be extended to a local scale-invariance [14, 15]. One of the simplest predictions of that theory is the explicit form of the two-time autoresponse function. It readsł

$$
R(t, s)=s^{-1-a} f_{R}(t, s), f_{R}(y)=f_{0} y^{1+a^{\prime}-\lambda_{R} / z}(y-1)^{-1-a^{\prime}}
$$

where $a^{\prime}$ is a new independent exponent and $f_{0}$ is a normalization constant. The independent existence of the exponent $a^{\prime}$ was recognized recently [17] for $z=2$ and we shall extend that argument to arbitrary $z$ in appendix A. Previous derivations of $f_{R}(y)[14,15]$ had assumed $a=a^{\prime}$ from the outset. The only known example with distinct exponents $a \neq a^{\prime}$ was the $1 D$ Glauber-Ising model at temperature $T=0$ and with initial power-law correlations of the form $\left\langle\sigma_{i} \sigma_{j}\right\rangle \sim|i-j|^{-\nu}$ with $\nu \geq 0$. The exact solution for $R(t, s)[18,19,20]$ is of the form (4) and one can read off $a=0$, $a^{\prime}=-\frac{1}{2}$ and $\lambda_{R}=1$ [17]. In appendix B, we give a second example and show that the treatment of phase-ordering in $d$ spatial dimensions at zero temperature in the spirit of the OJK-approximation $[21,22]$ leads to

$$
a_{\mathrm{OJK}}=\frac{d-1}{2}, \quad a_{\mathrm{OJK}}^{\prime}=\frac{d-2}{2}, \lambda_{R, \mathrm{OJK}}=\frac{d}{2}
$$

The same scaling function and hence (5) is also found from the gaussian approximation to phase-ordering kinetics [23].§

Eq. (4) with $a=a^{\prime}$ is perfectly reproduced in simple magnets undergoing coarsening after a quench to $T<T_{c}$, most notably the $2 D / 3 D$ Glauber-Ising model $[15,24]$, the $3 D \mathrm{XY}$ model [25] and in several exactly solvable systems [14, 27, 17].\| For critical quenches $T=T_{c}$, the agreement between numerical data and (4) with $a=a^{\prime}$ is almost perfect in the $2 D / 3 D$ Ising and XY models $[15,27,25,28]$, the $1 D$ contact process [29] and is exact in several exactly solvable models with $z=2[14,12,17]$. On the other hand, a second-order $\varepsilon$-expansion calculation from renormalized field-theory gives a small but systematic deviation with respect to eq. (4) $[9,30]$.

Of course it would be interesting to see whether a scaling description or even an extension to local scale-invariance might be applicable to glassy systems as well. Such a comparison may be far from straightforward, however. Indeed, a possible scaling behaviour of two-time correlators $C(t, s)$ and integrated responses $\int \mathrm{d} u R(t, u)$ have been discussed since a long time, both theoretically and experimentally, see $[1,3,6,4,31,8]$ and debates have arisen over several central issues of which we mention a few. First, ‡ Technically, this requires that the order parameter is a quasi-primary field under local scaletransformations [14]. This concept is the analogue of the one used in conformal field-theory [16].

$\S$ We observe that in both examples $a=a^{\prime}+\frac{1}{2}$ but it is still open to what extent this might be a general relationship.

|| A further extension of local scale-invariance with $z=2$ in $d$ spatial dimensions to a new type of conformal invariance in $d+2$ dimensions yields a prediction of $C(t, s)$ which is in agreement with numerical data in the $2 D$ Glauber-Ising model [26]. 
for glasses quenched to below their glass temperature, it is not entirely clear whether the growth law for $L(t)$ is algebraic or logarithmic [32]. Second, even if an asymptotic power-law scaling is accepted, there has been an intense debate on whether scaling occurs according to the so-called 'full ageing' scenario, that is in terms of the scaling variable $y=t / s$ (possibly with small logarithmic corrections) [33], or else if a 'subageing' scenario applies, with a scaling variable $\xi:=\left[t^{1-\mu}-s^{1-\mu}\right] /(1-\mu)$, where $\mu$ is a free parameter [31]. The usual power-law scaling is recovered in the $\mu \rightarrow 1$ limit, but in many experiments the data are fitted with values of $\mu$ as low as $\approx 0.8-0.9$. It has been suggested recently [34] that values of $\mu<1$ merely result from a quench to below the glass transition which is not yet sufficiently rapid, but the repetition of that experiment on other substances has not yet led to unambiguous conclusions [31]. Third, it is even no longer obvious that the commonly studied spin glass models really mimic sufficiently well the experimentally studied materials (in spite of well-established qualitative similarities [35]): recent simulations on $3 D / 4 D$ Ising and Heisenberg spin glasses provide evidence for cumulative ageing and rejuvenation phenomena in temperature cycling which are not observed in real spin glass materials [36].

In view of these many difficulties, it might be simpler to consider the behaviour of glassy systems from a different point of view. One rather works with a time-dependent (oscillating) magnetic field and studies simultaneously the dependence on time and on the imposed oscillation angular frequency $\omega$. For a harmonic magnetic field, it is common to consider the real and the imaginary part of the magnetic susceptibility

$$
\begin{aligned}
& \chi^{\prime}(\omega, t)=\int_{0}^{t} \mathrm{~d} u R(t, u) \cos (\omega(t-u)) \\
& \chi^{\prime \prime}(\omega, t)=\int_{0}^{t} \mathrm{~d} u R(t, u) \sin (\omega(t-u))
\end{aligned}
$$

where $R(t, s)$ is the linear response discussed above. In this setting, $1 / \omega$ provides the second time-scale, the natural scaling variable is $y=\omega t$ and the scaling regime should be reached in the limit $\omega \rightarrow 0$ and $t \rightarrow \infty$. In many experiments and simulations, one averages over at least one period of the oscillating field, see e.g. [35]. Then in a great variety of glass-forming substances quenched to below or near to their glass transition point one observes good but not always perfect evidence for an $\omega t$-scaling behaviour of the following form of the period-averaged dissipative (imaginary) part $[32,37]$

$$
\overline{\chi^{\prime \prime}}(\omega, t)=\chi_{\mathrm{st}}^{\prime \prime}(\omega)+\chi_{\text {age }}^{\prime \prime}(\omega, t), \quad \chi_{\text {age }}^{\prime \prime}(\omega, t) \simeq A_{\text {age }}^{\prime \prime}(\omega t)^{-b^{\prime \prime}}
$$

where $\chi_{\text {st }}^{\prime \prime}$ is thought of as a 'stationary' contribution while the ageing behaviour is described by $\chi_{\text {age }}^{\prime \prime}$. The amplitude $A_{\text {age }}^{\prime \prime}$ and the exponent $b^{\prime \prime}$ are obtained from fits to the experimental data but there does not seem to exist a relationship with the exponents $a, a^{\prime}, b, \lambda_{C, R}$ in the literature.

Similar scaling forms have been proposed for the dispersive (real) part $\overline{\chi^{\prime}}$ but in practice the imaginary part is usually easier to measure. It is usually thought that $b^{\prime}=b^{\prime \prime}$. 
In this paper, we shall try and see whether a relationship between the exponents $b^{\prime \prime}$ and $b^{\prime}$ of the alternating susceptibilities (6) and the other nonequilibrium exponents arising in the two-time observables can be found. Assuming the validity of local scaleinvariance for the relatively large values of $z$ (usually, $z \approx 5-7$ [8]) found in many studies of spin glasses and hence the explicit form (4) for the autoresponse function, 9 we shall show that for large times

$$
\begin{aligned}
& \chi^{\prime \prime}(\omega, t)=\chi_{1}^{\prime \prime}(\omega)+\omega^{a} \chi_{2}^{\prime \prime}(\omega t)+\mathrm{O}\left(t^{-\lambda_{R} / z}\right) \\
& \chi^{\prime}(\omega, t)=\chi_{1}^{\prime}(\omega)+\omega^{a} \chi_{2}^{\prime}(\omega t)+\mathrm{O}\left(t^{-\lambda_{R} / z}\right)
\end{aligned}
$$

and we shall calculate the scaling functions $\chi_{2}^{\prime \prime}$ and $\chi_{2}^{\prime}$ explicitly in section 2 . For the asymptotics of these scaling functions we expect $\chi_{2}^{\prime}(y) \sim y^{-b^{\prime}}$ and $\chi_{2}^{\prime \prime}(y) \sim y^{-b^{\prime \prime}}$ for $y \gg 1$ and obtain the relation

$$
b^{\prime}=b^{\prime \prime}=a-a^{\prime}
$$

This should be compared to the experimentally found scaling (7) of $\chi_{\text {age }}^{\prime \prime}(\omega t)$ and similarly for $\chi_{\text {age }}^{\prime}(\omega t)$. In section 3 we compare these results with Monte-Carlo data from the $3 D$ Ising spin glass with a binary distribution of the couplings and discuss to what extent the scaling relations (9) and the explicit scaling functions agree with existing experimental data. We conclude in section 4. In appendix A we derive eq. (4) from (an extension of) local scale-invariance and in appendix B we revisit the OJK-approximation of coarsening kinetics and derive (5).

\section{Scaling of the alternating susceptibility}

We now analyze the frequency-dependent scaling of the alternating susceptibility. For notational simplicity, we concentrate first on $\chi^{\prime \prime}(\omega, t)$ as given by eq. (6). In order to make the scaling behaviour explicit, we must convert this into a more convenient form which can be done as follows [38]. We observe that the time difference $\tau=t-u$ plays a central rôle since depending on its value either an equilibrium behaviour or else an ageing behaviour is obtained. Specifically, it can be shown [39] that there is a time-scale $t_{p} \sim t^{\zeta}$ with $0<\zeta<1$ on which the transition between the two regimes occurs such that $R(t, s) \simeq R_{\mathrm{eq}}(t-s)$ for $t-s \lesssim t_{p}$ and $R(t, s)=s^{-1-a} f_{R}(t / s)$ as given in eq. (4) for $t-s \gtrsim t_{p} .{ }^{+}$In addition, one measures for $u \approx t$ the response with respect to a change in the initial conditions and then instead of $(4)$ one expects $R \approx R_{\mathrm{ini}}(t) \sim t^{-\lambda_{R} / z}$ [2]. We therefore must introduce a further time-scale $t_{\varepsilon}$ such that $t-t_{\varepsilon}=\mathrm{O}(1)$. Changing variables and then splitting the integral into three terms corresponding to these three

I Our recent study of the critical $3 D / 4 D$ Ising spin glass [13] showed that the form of the measured thermoremanent magnetization $\rho(t, s)=\int_{0}^{s} \mathrm{~d} u R(t, u)$ agrees with the prediction of local scale-invariance for $t / s \lesssim 20$, which is also in the sector encountered in $\omega t$-scaling.

+ Explicitly, $\zeta=4 /(d+2)$ in the $d$-dimensional spherical model [39]. 
regimes, we have

$$
\begin{aligned}
\chi^{\prime \prime}(\omega, t)=\int_{0}^{t} \mathrm{~d} \tau R(t, t-\tau) \sin \omega \tau \\
=\int_{0}^{t_{p}} \mathrm{~d} \tau R(t, t-\tau) \sin \omega \tau+\int_{t_{p}}^{t_{\varepsilon}} \mathrm{d} \tau R(t, t-\tau) \sin \omega \tau \\
\quad+\int_{t_{\varepsilon}}^{t} \mathrm{~d} \tau R(t, t-\tau) \sin \omega \tau \\
\simeq \quad \int_{0}^{t_{p}} \mathrm{~d} \tau R_{\text {eq }}(\tau) \sin \omega \tau+t^{-a} \int_{t_{p} / t}^{t_{\varepsilon} / t} \mathrm{~d} v f_{R}\left(\frac{1}{1-v}\right) \frac{\sin \omega t v}{(1-v)^{1+a}} \\
\quad+t^{-\lambda_{R} / z} \int_{t_{\varepsilon}}^{t} \mathrm{~d} \tau c_{0} \sin \omega \tau \\
=\chi_{1}^{\prime \prime}(\omega)+t^{-a} \int_{0}^{1} \mathrm{~d} v f_{R}\left(\frac{1}{1-v}\right) \frac{\sin \omega t v}{(1-v)^{1+a}}+\mathrm{O}\left(t^{-\lambda_{R} / z}\right)
\end{aligned}
$$

In the third line, we used the asymptotic forms of $R(t, s)$ as described above. This means that the cross-over between the equilibrium and the ageing regimes is assumed to be very rapid. In the last line, we restricted ourselves to the long-time limit $t \rightarrow \infty$. Here, the function $\chi_{1}^{\prime \prime}(\omega)$ merely depends on the equilibrium form of the linear response $R_{\text {eq }}(t, s)$.

In this way (analogously for $\chi^{\prime}$ ) the scaling form (8) is obtained. This derivation also shows that the often-found stationary term in the integrated response $[3,40,4,31$, $32,8,41,34,37]$ does not require the separation of a similar 'stationary' part in the response function $R(t, s)$ itself.

We now analyze the second term in the above expression for $\chi^{\prime \prime}$. Using the explicit form (4) for the scaling function $f_{R}$, we have

$$
\chi^{\prime \prime}(\omega, t)=\chi_{1}^{\prime \prime}(\omega)+f_{0} t^{-a} S+\mathrm{O}\left(t^{-\lambda_{R} / z}\right)
$$

where expansion of the sine followed by term-wise integration gives

$$
\begin{aligned}
S: & \int_{0}^{1} \mathrm{~d} v(1-v)^{-1-a+\lambda_{R} / z} v^{-1-a^{\prime}} \sin (\omega t v) \\
= & \sum_{n=0}^{\infty} \frac{(-1)^{n}}{(2 n+1) !} B\left(2 n+1-a^{\prime}, \frac{\lambda_{R}}{z}-a\right)(\omega t)^{2 n+1} \\
= & B\left(1-a^{\prime}, \frac{\lambda_{R}}{z}-a\right) \omega t \\
& \times{ }_{2} F_{3}\left(\frac{1-a^{\prime}}{2}, \frac{2-a^{\prime}}{2} ; \frac{3}{2}, \frac{1-a-a^{\prime}}{2}+\frac{\lambda_{R}}{2 z}, \frac{2-a-a^{\prime}}{2}+\frac{\lambda_{R}}{2 z} ;-\frac{\omega^{2} t^{2}}{4}\right)
\end{aligned}
$$

where ${ }_{2} F_{3}$ is a hypergeometric function,

$$
B(z, w)=\frac{\Gamma(z) \Gamma(w)}{\Gamma(z+w)}=\int_{0}^{1} \mathrm{~d} u u^{z-1}(1-u)^{w-1}
$$


is Euler's betafunction and the identity $\Gamma(2 z)=2^{2 z-1} \Gamma(z) \Gamma(z+1 / 2) / \sqrt{\pi}$ was also used.

We proceed to extract the leading behaviour for large values of the scaling variable $y=\omega t$. Recall first the asymptotic expansion for $x \rightarrow+\infty$ [42]

$$
\begin{aligned}
{ }_{2} F_{3}(a, b ; c, d, e ;-x) & \simeq\left[A x^{-a}+B x^{-b}+C x^{\Delta / 2} \cos \left(2 \sqrt{x}+\frac{\pi}{2} \Delta\right)\right] \\
& \times\left(1+\mathrm{O}\left(x^{-1}\right)\right)
\end{aligned}
$$

with $\Delta=a+b-c-d-e+\frac{1}{2}$ and where the constants $A, B, C$ are given by

$$
\begin{aligned}
& A=\frac{\Gamma(c) \Gamma(d) \Gamma(e)}{\Gamma(b)} \frac{\Gamma(b-a)}{\Gamma(c-a) \Gamma(d-a) \Gamma(e-a)} \\
& B=\frac{\Gamma(c) \Gamma(d) \Gamma(e)}{\Gamma(a)} \frac{\Gamma(a-b)}{\Gamma(c-b) \Gamma(d-b) \Gamma(e-b)} \\
& C=\frac{\Gamma(c) \Gamma(d) \Gamma(e)}{\sqrt{\pi} \Gamma(a) \Gamma(b)}
\end{aligned}
$$

Inserting this into the expression for $S$, we find

$$
S \simeq \bar{A}(\omega t)^{a^{\prime}-a}+\bar{B}(\omega t)^{a^{\prime}-a-1}+\bar{C}(\omega t)^{-\lambda_{R} / z} \sin \left(\omega t+\frac{\pi}{2}\left(a-\lambda_{R} / z\right)\right)
$$

where the constants $\bar{A}, \bar{B}, \bar{C}$ are proportional to $A, B, C$. The second term in (16) is always non-leading. The other two terms, however, will determine the functional form of the scaling function for $y=\omega t$ sufficiently large. The treatment of $\chi^{\prime}$ is analogous.

We can summarize the content of this section by listing the scaling functions which occur in (8), together with their leading behaviour as $y \rightarrow \infty$

$$
\begin{aligned}
& \chi_{2}^{\prime \prime}(y)=f_{0} B\left(1-a^{\prime}, \frac{\lambda_{R}}{z}-a\right) y^{1-a} \\
& \times{ }_{2} F_{3}\left(\frac{1-a^{\prime}}{2}, \frac{2-a^{\prime}}{2} ; \frac{3}{2}, \frac{1-a-a^{\prime}}{2}+\frac{\lambda_{R}}{2 z}, \frac{2-a-a^{\prime}}{2}+\frac{\lambda_{R}}{2 z} ;-\frac{y^{2}}{4}\right) \\
& \simeq f_{0} \frac{\pi}{2}\left[\cos \left(\frac{\pi a^{\prime}}{2}\right) \Gamma\left(1+a^{\prime}\right)\right]^{-1} y^{a^{\prime}-a}+f_{0} \Gamma\left(\frac{\lambda_{R}}{z}-a\right) y^{-\lambda_{R} / z} \sin \left(y+\frac{\pi}{2}\left[a-\lambda_{R} / z\right]\right) \\
& \chi_{2}^{\prime}(y)=f_{0} B\left(-a^{\prime}, \frac{\lambda_{R}}{z}-a\right) y^{-a} \\
& \times{ }_{2} F_{3}\left(\frac{-a^{\prime}}{2}, \frac{1-a^{\prime}}{2} ; \frac{1}{2}, \frac{-a-a^{\prime}}{2}+\frac{\lambda_{R}}{2 z}, \frac{1-a-a^{\prime}}{2}+\frac{\lambda_{R}}{2 z} ;-\frac{y^{2}}{4}\right) \\
& \simeq-f_{0} \frac{\pi}{2}\left[\sin \left(\frac{\pi a^{\prime}}{2}\right) \Gamma\left(1+a^{\prime}\right)\right]^{-1} y^{a^{\prime}-a}+f_{0} \Gamma\left(\frac{\lambda_{R}}{z}-a\right) y^{-\lambda_{R} / z} \cos \left(y+\frac{\pi}{2}\left[a-\lambda_{R} / z\right]\right)
\end{aligned}
$$

We see that there appear terms which decrease monotonously with $y$ but that there are also oscillating terms. They are described by different exponents and must be extracted by a different experimental setup. The oscillating terms follow the oscillations of the external field and the decrease of the oscillation amplitude gives a direct access to the exponent $\lambda_{R} / z$. On the other hand, in many experiments the data are averaged over one or several periods of the external field. For $y$ sufficiently large, the contribution 
of the oscillating term in eqs. $(17,18)$ vanishes after averaging and then only a simple algebraic component remains, which permits to extract the exponent $a-a^{\prime}$. For periodaveraged data or else if $\lambda_{R} / z \geq a-a^{\prime}$, the leading behaviour for large arguments is

$$
\chi_{2}^{\prime}(y) \sim \chi_{2}^{\prime \prime}(y) \sim y^{a^{\prime}-a}
$$

and the scaling relations (9) follow.

\section{Tests}

\subsection{Numerical simulations}

We now compare the theory of the alternating susceptibility developed in the previous section with numerical simulations performed on the critical three-dimensional Ising spin glass. The Hamiltonian of the model is given by

$$
\mathcal{H}=-\sum_{(\boldsymbol{i}, \boldsymbol{j})} J_{\boldsymbol{i}, \boldsymbol{j}} \sigma_{\boldsymbol{i}} \sigma_{\boldsymbol{j}}
$$

where the nearest-neighbour couplings $J_{\boldsymbol{i}, \boldsymbol{j}}$ are random variables taken from a binary distribution, i.e. $J_{\boldsymbol{i}, \boldsymbol{j}}= \pm 1$ with equal probability. The classical Ising spins $\sigma_{\boldsymbol{i}}= \pm 1$ characterize the local magnetization at the sites $\boldsymbol{i}$ of a simple cubic lattice. In the past, numerical studies $[43,44]$ investigated the static critical properties of this model which undergoes a continuous phase transition at the temperature $T_{c} \approx 1.19\left(\right.$ setting $\left.k_{B}=1\right)$. Recently, we studied the ageing behaviour of this critical spin glass [13]. There we found clear evidence of a power-law scaling in the two-time correlation and integrated response functions. Here we shall need the exponent estimates $a=0.060(4)$ and $\lambda_{R} / z=0.38(2)$ obtained from a scaling analysis of the thermoremanent magnetization.

In order to study the alternating susceptibility far from equilibrium we prepared the system in an uncorrelated initial state (corresponding to an infinite initial temperature) before quenching it to the critical point at time $t=0$. At the same time an external oscillating magnetic field

$$
h(t)=h_{0} \cos \omega t
$$

was switched on, with it's amplitude fixed at $h_{0}=0.05$ which is well inside the linearresponse regime. We consider different values of the angular frequency $\omega=2 \pi / p$ with $p$ ranging from 50 to 1600 . Typically, systems containing $50^{3}$ spins were simulated.

Numerically, the in-phase and the out-of-phase susceptibilities are given by the expressions [45]

$$
\begin{aligned}
& \chi^{\prime \prime}(\omega, t)=m(t) \sin \omega t \\
& \chi^{\prime}(\omega, t)=m(t) \cos \omega t
\end{aligned}
$$




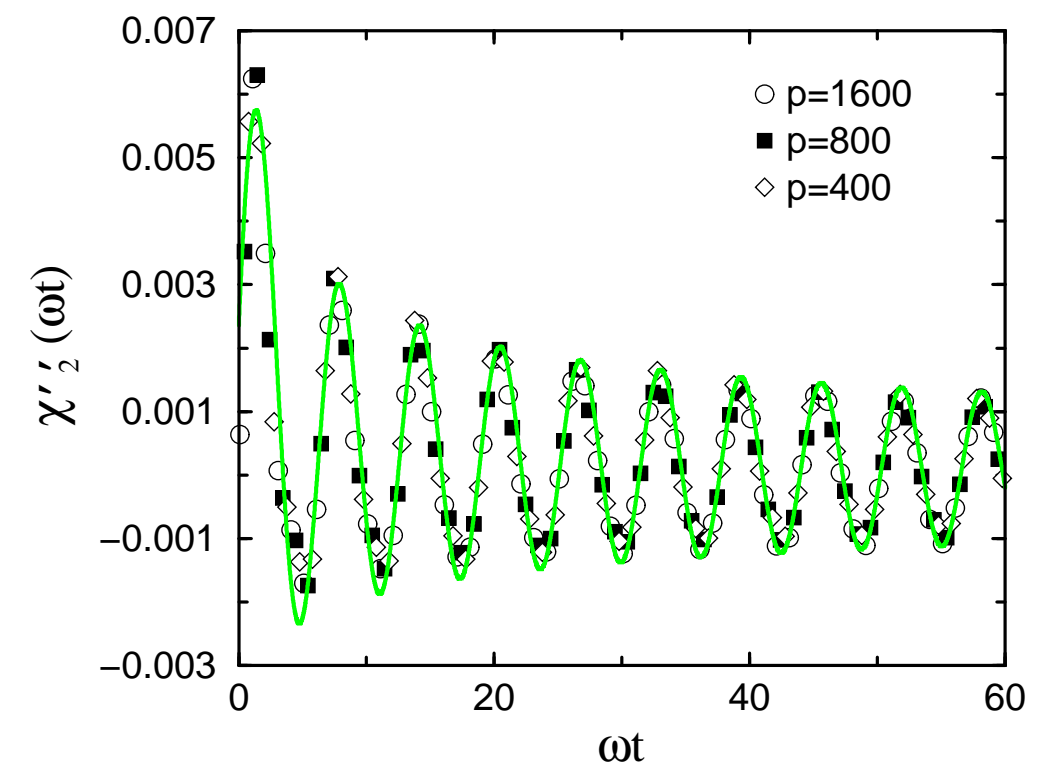

Figure 1. Scaling of the dissipative part $\chi_{2}^{\prime \prime}(\omega t)$ of the alternating susceptibility as function of the scaling variable $\omega t$ for different angular frequencies $\omega=2 \pi / p$ with $p=1600,800$, and 400 . The full curve is the theoretical prediction (17) with $f_{0}=0.002$ and $a^{\prime}=-0.70$ but which has also been shifted horizontally by $y \rightarrow y+\Delta y$, with $\Delta y=-0.45$, see text. Statistical error bars are smaller than the symbol sizes.

with $m(t)=\sum_{\boldsymbol{i}} \sigma_{\boldsymbol{i}}(t)$. In order to access the scaling parts of $\chi^{\prime \prime}$ and of $\chi^{\prime}$, we must first subtract the equilibrium parts $\chi_{1}^{\prime \prime}$ and $\chi_{1}^{\prime}$. We therefore carried out longer runs where we let the system equilibrate for typically a few ten thousand time steps before switching on the oscillating field. When no change in the amplitudes of the alternating susceptibilities were observed, we identified these data with the equilibrium parts $\chi_{1}^{\prime \prime}$ and $\chi_{1}^{\prime}$. The ageing parts of $\chi^{\prime \prime}$ and of $\chi^{\prime}$ discussed in the following result from averaging over 2500 different runs with different couplings, different initial states and different realizations of the thermal noise.

In figures 1 and 2 we test the expected scaling behaviour of the ageing part, see eq. (7)

$$
\chi_{2}^{\prime \prime}=\chi_{2}^{\prime \prime}(\omega t) \quad \text { and } \quad \chi_{2}^{\prime}=\chi_{2}^{\prime}(\omega t) .
$$

For the larger values of $p$, corresponding to the smaller values of $\omega$, we observe a very good data collapse for both quantities, which furnishes clear evidence in favour of a power-law scaling at $T=T_{c}$. Here we used the value $a=0.060(4)$ determined previously from the decay of the thermoremanent magnetization [13]. For smaller values of $p$, the collapse is less good, which presumably means that for the corresponding values of $\omega$ the dynamical scaling regime is not yet reached.

The data shown in these two figures can in principle be compared directly with the analytical predictions (17) and (18). We point out, however, that the positions of 


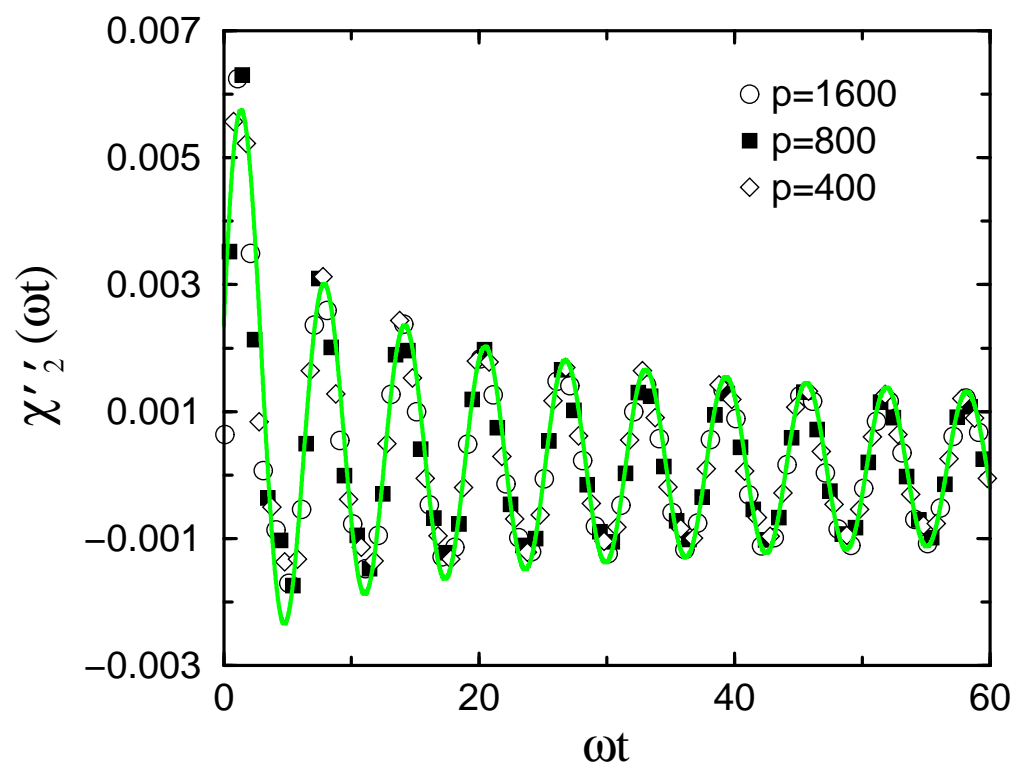

Figure 2. The same as in Figure 1, but now for the dispersive part $\chi_{2}^{\prime}(\omega t)$. The full curve is the shifted theoretical prediction (18) with $f_{0}=0.002$ and $a^{\prime}=-0.70$.

maxima of $\chi_{2}^{\prime \prime}$ and $\chi_{2}^{\prime}$ are shifted by the amount $\Delta y \approx-0.45$ when compared with the positions obtained in the analytical treatment of the previous section. The origin of this small shift is not completely clear to us. It is possible, however, that the cross-over between the equilibrium and the ageing regimes is not almost instantaneous in contrast to what we assumed in the derivation of eq. (10). In any case, we shifted the scaling variable in the analytical curves correspondingly, in order to make a comparison between analytical prediction and numerical data possible.

As both $a$ and $\lambda_{R} / z$ are known from our earlier investigation [13] the only free parameters in this comparison are the amplitude $f_{0}$ and the exponent $a^{\prime}$. Our final estimates are

$$
a^{\prime}=-0.70(3), f_{0}=0.00203(1)
$$

and the fit is compared to the data in the figures. These values (23) of the parameters describe consistently both $\chi_{2}^{\prime \prime}$ and $\chi_{2}^{\prime}$. Although some discrepancies are observed for small values of $y=\omega t$, the overall agreement between the simulation and the shifted theoretical prediction is very good. The numerical data therefore support the scaling approach presented in the previous section.

\subsection{Comparison with experiments}

We now turn to a discussion of existing experimental results on the scaling of the alternating susceptibility.

A detailed discussion of a possible scaling of the alternating susceptibility was 
presented by Suzuki and Suzuki for the short-ranged Ising spin glass $\mathrm{Cu}_{0.5} \mathrm{Co}_{0.5} \mathrm{Cl}_{2^{-}}$ $\mathrm{FeCl}_{3}$ graphite bi-intercalation compound [37]. After a rapid quench to below the glass temperature $T_{g}=3.92(11) \mathrm{K}$, they measure $\chi^{\prime \prime}(\omega, t)$ for fixed $\omega$ and find that their (period-averaged) data are well-fitted by the power-law

$$
\overline{\chi^{\prime \prime}}(\omega, t)=\chi_{0}^{\prime \prime}(\omega)+A^{\prime \prime}(\omega) t^{-b^{\prime \prime}}
$$

While the fitted exponent $b^{\prime \prime}$ depends only slightly on $\omega$, they further show evidence for a power-law $A^{\prime \prime}(\omega)=A_{0}^{\prime \prime} \omega^{-\mu^{\prime \prime}}$ and find that "... the value of $\mu^{\prime \prime}$ is almost the same as that of $b^{\prime \prime} "[37]$. In this way, they arrive at the $\omega t$-scaling form

$$
\overline{\chi^{\prime \prime}}(\omega, t)=\chi_{0}^{\prime \prime}(\omega)+A_{0}^{\prime \prime}(\omega t)^{-b^{\prime \prime}}
$$

and a similar form for $\overline{\chi^{\prime}}(\omega, t)$ where "..$b^{\prime}$ and $b^{\prime \prime}$ are of the same order at the same temperature" [37]. Experimentally measured values of the exponent $b^{\prime \prime}$ (and also $b^{\prime}$ ) of some materials are collected in table 1 . We now compare the experimental results of Suzuki and Suzuki [37] with the theoretical scaling form (8). First, the experimental evidence for a pure $\omega t$-scaling indicates that the exponent $a$ must indeed be very small. Second, when considering the leading behaviour for $y=\omega t$ large (their data go up to $\left.y \lesssim 10^{6}[37]\right)$ and recalling that the experimental data are averaged over at least one period of the external field, we can read off

$$
b^{\prime \prime}=a-a^{\prime}, \quad \mu^{\prime \prime}=-a^{\prime}
$$

and the observed [37] near equality $b^{\prime \prime} \approx \mu^{\prime \prime}$ is again consistent with $a$ being negligibly small. Third, the available data are consistent with the theoretically requested relation $b^{\prime}=b^{\prime \prime}$. Forth, the reason for the experimentally observed sudden jump in $b^{\prime}$ and $b^{\prime \prime}$ for smaller $T$ is not yet understood but we mention that a similar phenomenon also occurs in certain relaxor ferroelectrics $[40,46]$.

Similar values of $b^{\prime \prime}$ were observed for several other materials, quite independently of the precise physical nature as can be seen from table 1, but the errors are still too large to permit a discussion of the universality of the exponents. However, the experimental data are in many of these systems at least as well described by a logarithmic scaling as expected from the droplet theory [48, 32, 49]. Furthermore, in several systems also strong deviations from a simple $\omega t$-scaling were found, see [46]. Finally, we mention that in systems like $\beta$-hydroquinol-clathrate [41] or even simple liquids like glycerol [50] a power-law dependence of the form $\chi_{\text {age }}^{\prime \prime} \sim t^{-a}$ or $\chi_{\text {age }}^{\prime} \sim t^{-a}$ was observed. All in all, it is not yet completely understood what precise conditions are needed such that a clear power-law scaling in $\omega t$ can be observed.

Lastly, we see that the value $b^{\prime \prime} \simeq 0.7$ obtained from the critical $3 D$ Ising spin glass with binary disorder is very far from the values $b^{\prime \prime} \approx 0.1-0.2$ found experimentally. 
Table 1. Measured values of the exponents $b^{\prime \prime}$ and $b^{\prime}$ in several glassy materials, using the scaling form (7). Here $T_{g}$ stands for the glass transition temperature and $T$ is the temperature where the data were taken. For $\mathrm{Fe}_{0.5} \mathrm{Mn}_{0.5} \mathrm{TiO}_{3}$ and $\mathrm{CdCr}_{1.7} \mathrm{In}_{0.3} \mathrm{~S}_{4}$ the relation $b^{\prime}=b^{\prime \prime}$ was assumed.

\begin{tabular}{|c|c|c|c|c|c|c|}
\hline \multirow{2}{*}{\begin{tabular}{l}
\multicolumn{1}{c}{ Material } \\
$\mathrm{Cu}_{0.5} \mathrm{Co}_{0.5} \mathrm{Cl}_{2}-\mathrm{FeCl}_{3}$ \\
$-\mathrm{GBIC}$
\end{tabular}} & $T_{g}[\mathrm{~K}]$ & $T[\mathrm{~K}]$ & $b^{\prime \prime}$ & $b^{\prime}$ & Ref. & \\
\hline & $3.92(11)$ & $\begin{array}{l}3.25 \\
3.5 \\
3.75 \\
3.85 \\
3.95\end{array}$ & $\begin{array}{l}0.01(4) \\
0.017(32) \\
0.16(3) \\
0.15(3) \\
0.16(4)\end{array}$ & $\begin{array}{l}0.08(3) \\
0.05(2) \\
0.20(2) \\
0.20(2)\end{array}$ & {$[37]$} & Ising spin glass \\
\hline $\mathrm{Fe}_{0.5} \mathrm{Mn}_{0.5} \mathrm{TiO}_{3}$ & 20.7 & $\begin{array}{l}15 \\
19\end{array}$ & $\begin{array}{l}0.14(3) \\
0.14(3)\end{array}$ & & {$[49,48]$} & Ising $\mathrm{sI}$ \\
\hline $\mathrm{CdCr}_{1.7} \mathrm{In}_{0.3} \mathrm{~S}_{4}$ & 16.7 & $\begin{array}{l}12 \\
14\end{array}$ & $\begin{array}{l}0.18(3) \\
0.18(3)\end{array}$ & & {$[49,48]$} & Heisenberg spin glass \\
\hline $\begin{array}{rr}\mathrm{CdCr}_{2 x} \operatorname{In}_{2-2 x} \mathrm{~S}_{4} & x=0.95 \\
& x=0.90 \\
\end{array}$ & $\begin{array}{l}70 \\
50 \\
\end{array}$ & $\begin{array}{l}8 \\
67 \\
42 \\
\end{array}$ & $\begin{array}{l}0.2 \\
0.2 \\
0.20 \\
\end{array}$ & & $\begin{array}{l}51,48] \\
{[47,48]} \\
\end{array}$ & disordered ferromagnet \\
\hline $\mathrm{Pb}\left(\mathrm{Mg}_{1 / 3} \mathrm{Nb}_{2 / 3}\right) \mathrm{O}_{3}$ & $\sim 220$ & $\lesssim 220$ & 0.17 & & {$[40]$} & relaxor ferroelectric \\
\hline
\end{tabular}

\section{Conclusions and discussion}

The objective of this work has been to investigate to what extent the ageing behaviour of the dispersive and dissipative parts of the alternating susceptibility in glassy systems may be described in terms of some simple ideas borrowed from the ageing of simple magnets without disorder. These are

(i) the clear separation of the stationary and the ageing regimes, which goes into the derivation of eq. (10).

(ii) the hypothesis of a single essential length scale $L(t) \sim t^{1 / z}$ growing algebraically in time.

(iii) the extension of this dynamical scaling to a local scale-invariance which leads to the simple form eq. (4) for the two-time response function $R(t, s)$.

Taking these assumptions as working hypothesis, our results are as follows:

(i) The often-studied simple $\omega t$-scaling (7) in $\chi^{\prime \prime}$ and $\chi^{\prime}$ should be slightly generalized to the scaling forms (8) or equivalently

$$
\chi^{\prime \prime}(\omega, t)=\chi_{1}^{\prime \prime}(\omega)+t^{-a} \bar{\chi}_{2}^{\prime \prime}(\omega t) \quad \text { with } \quad \bar{\chi}_{2}^{\prime \prime}(y)=y^{a} \chi_{2}^{\prime \prime}(y)
$$

and similarly for $\chi^{\prime}$. The smallness of the exponent a makes it conceivable that the slight 'subageing' found in many experiments might be taken into account this 
way.* Tests of this idea in real materials would be welcome.

(ii) The exponents $b^{\prime}$ and $b^{\prime \prime}$ defined from $\chi_{2}^{\prime}(y) \sim y^{-b^{\prime}}$ and $\chi_{2}^{\prime \prime}(y) \sim y^{-b^{\prime \prime}}$ satisfy the scaling relation

$$
b^{\prime}=b^{\prime \prime}=a-a^{\prime}
$$

which hence relates exponents found in an oscillating field to those describing the two-time response $R(t, s)$. Since $a^{\prime}$ appears to be a new, independent exponent, this suggests that the exponent $b^{\prime}=b^{\prime \prime}$ should be independent of the exponents $a, b, \lambda_{C} / z, \lambda_{R} / z$ habitually used in describing ageing. This question could be addressed, independently of the hypothesis of local scale-invariance and the form (4), through field-theory methods as developped in [52].

(iii) When testing the explicit dispersive and dissipative scaling functions (17) and (18) on the $3 D$ critical Ising spin glass with binary disorder, we found that the form of both is reproduced by the data for the following exponents

$$
a=0.060(4), a^{\prime}=-0.70(3), \frac{\lambda_{R}}{z}=0.38(2)
$$

(and with a normalization constant $f_{0} \simeq 0.002$ ) where we took over the values of $a$ and $\lambda_{R} / z$ from our earlier analysis of $R(t, s)$ in this model [13]. We stress that this agreement is only found if the curves (17) and (18) are shifted $y \rightarrow y+\Delta y$ by a constant $\Delta y \simeq-0.45$. Even then, for small values of $y$ the simulated scaling functions show a small but systematic deviation from the theoretical prediction which remains to be understood.

The origin of this shift is unknown to us, but it might be due to a rather slow cross-over between the stationary and the ageing regimes which is not captured by eq. (10). Understanding this point is an important open problem.

We think it is remarkable that the ageing dynamics of a system as complicated as a critical spin glass can be captured by our relatively simple hypothesis of a dynamical symmetry.

(iv) The value $b^{\prime \prime} \simeq 0.7$ we found in the $3 D$ Ising spin glass (with binary disorder) is far from the values obtained in experiments, see table $1 . \#$ If the present result should be confirmed, it might furnish a further hint towards a fundamental difference between simple spin glass models and experimentally realized glassy systems [36].

On the other hand, the theoretically predicted relation $b^{\prime}=b^{\prime \prime}$ is fully consistent with the available experimental results.

Acknowledgements: We thank G.F. Mazenko for helpful correspondence and O. Petracic for a useful discussion. This work was supported by the BayerischFranzösisches Hochschulzentrum (BFHZ) and by CINES Montpellier (projet pmn2095).

* Working with (27) rather than with (8) has the advantage that for $y \gg 1$ the first term in the asymptotic expansion only depends on the exponent $a^{\prime}$, while the second one only depends on $a-\lambda_{R} / z$. $\sharp$ Our previous investigation of the thermoremanent magnetization [13] did not permit us to determine the value of $a^{\prime}$. 
MP acknowledges the support by the Deutsche Forschungsgemeinschaft through grant no. PL 323/2.

\section{Appendix A. The autoresponse function and extended local scale-invariance}

Local scale-transformations are extensions of the dynamical scale-transformation $\boldsymbol{r} \mapsto$ $b \boldsymbol{r}, t \mapsto b^{z} t$ of space-time towards variable rescaling factors $b=b(t, \boldsymbol{r})$ such that conformal transformation in time $t \mapsto(\alpha t+\beta) /(\gamma t+\delta)$ with $\alpha \delta-\beta \gamma=1$ are maintained [14]. For any given value of $z$, such infinitesimal transformations have been explicitly constructed and shown to furnish a dynamical symmetry of the generalized diffusion equation $\left(\partial_{t}+\partial_{r}^{z}\right) \psi(t, r)=0$. Here we are interested in applications to ageing behaviour of the autoresponse function

$$
R(t, s)=\left.\frac{\delta\langle\phi(t, \boldsymbol{r})\rangle}{\delta h(s, \boldsymbol{r})}\right|_{h=0}=\langle\phi(t, \boldsymbol{r}) \widetilde{\phi}(s, \boldsymbol{r})\rangle
$$

where $\phi$ is the order parameter, $h$ the conjugate magnetic field and $\widetilde{\phi}$ the associate response field in the context of the Martin-Siggia-Rose theory, see e.g. [53]. Therefore, we have to restrict to the subalgebra with time-translations excluded [14, 15]. Since space translations are included in the set of local scale-transformations, the scaling of $R(t, s)$ will be entirely given in terms of merely two generators which we write as

$$
X_{0}=-t \partial_{t}-\frac{x}{z}, X_{1}=-t^{2} \partial_{t}-\frac{2}{z}(x+\xi) t
$$

where $x$ is the scaling dimensions of the field on which the generators $X_{n}$ act. We have observed earlier [17] in the special case $z=2$ that a further constant $\xi$ can be introduced without changing the commutator relations of the subalgebra of local-scale invariance under consideration and now extend this to arbitrary values of $z$. Previous treatments of the question had admitted $\xi=\widetilde{\xi}=0$ from the outset. The covariance of $R(t, s)$ is now expressed as usual $[14,15]$

$$
\begin{aligned}
& X_{0} R=\left(-t \partial_{t}-s \partial_{s}-\frac{x}{z}-\frac{\widetilde{x}}{z}\right) R(t, s)=0 \\
& X_{1} R=\left(-t^{2} \partial_{t}-s^{2} \partial_{s}-\frac{2}{z}(x+\xi) t-\frac{2}{z}(\widetilde{x}+\widetilde{\xi}) s\right) R(t, s)=0
\end{aligned}
$$

where $\widetilde{x}$ and $\widetilde{\xi}$ refer to the response field $\widetilde{\phi}$. To solve these, change variables into $u=t-s$ and $v=t / s$. Then, with $R(t, s)=\bar{R}(u, v)$

$$
\begin{array}{ll}
\left(u \partial_{u}+\frac{x+\widetilde{x}}{z}\right) \bar{R}(u, v) & =0 \\
u\left(v \partial_{v}+\frac{v}{v-1} \frac{x-\widetilde{x}+2 \xi}{z}+\frac{1}{v-1} \frac{\widetilde{x}-x+2 \widetilde{\xi}}{z}\right) \bar{R}(u, v) & =0
\end{array}
$$


The solution to these is found in factorized form $\bar{R}(u, v)=f(u) g(v)$ and we find, after having returned to the variables $t$ and $s$

$$
R(t, s)=r_{0} s^{-1-a}\left(\frac{t}{s}-1\right)^{-1-a^{\prime}}\left(\frac{t}{s}\right)^{1+a^{\prime}-\lambda_{R} / z}
$$

where

$$
\begin{aligned}
1+a & =\frac{x+\widetilde{x}}{z} \\
1+a^{\prime} & =\frac{x+\widetilde{x}+2 \xi+2 \widetilde{\xi}}{z} \\
\lambda_{R} & =2(x+\xi)
\end{aligned}
$$

which is the form (4) stated in the text. We finally observe that if $\xi+\widetilde{\xi}=0$, we recover indeed $a=a^{\prime}$.

\section{Appendix B. On the Ohta-Jasnow-Kawasaki approximation}

We briefly present the derivation of the scaling of the response function $R(t, s)$ in a simple analytically tractable scheme which is close in spirit to the Ohta-Jasnow-Kawasaki (OJK) approximation. Following the ideas of Berthier, Barrat and Kurchan [21] and of Mazenko [22] which in turn are based on a calculation by Bray [54], one considers the integrated response in the zero-field cooled protocol. When perturbing the system by a spatially random field $h$ of magnitude $h_{0}$, the zero-field-cooled (ZFC) susceptibility reads $[21,54]$

$$
\chi(t, s)=\frac{\langle h(\boldsymbol{r}) \phi(t, \boldsymbol{r})\rangle}{h_{0}^{2}} \simeq \sqrt{\frac{2}{\pi}} \frac{\langle h(\boldsymbol{r}) m(t, \boldsymbol{r})\rangle}{h_{0}^{2} \sqrt{\left\langle m^{2}\right\rangle}}
$$

where $\phi$ is the order parameter and it is assumed that for late times, one can approximate $\phi \sim \operatorname{sign}(m)$. At zero temperature, the auxiliary field $m$ should satisfy the equation of motion

$$
\frac{\partial m}{\partial t}=\nabla^{2} m-n_{a} n_{b} \nabla_{a} \nabla_{b} m+h|\nabla m| .
$$

In the spirit of the OJK-approximation, one makes [54,2] the simplifications $n_{a} n_{b} \rightarrow$ $\delta_{a b} / d$ (circular average) and $|\nabla m| \rightarrow\left\langle(\nabla m)^{2}\right\rangle^{1 / 2}$. The equation of motion then becomes $[21,54] \partial_{t} m=D \nabla^{2} m+h\left\langle(\nabla m)^{2}\right\rangle^{1 / 2}$ with $D=(d-1) / d$ and assuming the fields $m$ and $h$ to be gaussian, it is found that [21, eqns. $(19,20)]$

$$
\chi(t, s)=\int_{s}^{t} \mathrm{~d} u \frac{(D t)^{d / 4}}{(D u)^{(d+2) / 4}} \int_{\mathbb{R}^{d}} \mathrm{~d} \boldsymbol{k} e^{-k^{2} D(t-u)}
$$

which is the starting point of our analysis. 
Performing the integration in $\boldsymbol{k}$-space, one obtains [22]

$$
\chi(t, s)=\text { cste. } \int_{s}^{t} \mathrm{~d} u u^{-(d+1) / 2}\left(\frac{t}{u}\right)^{d / 4}\left(\frac{t}{u}-1\right)^{-d / 2} \stackrel{!}{=} \int_{s}^{t} \mathrm{~d} u R(t, u) .
$$

This integral becomes singular near the upper limit $u \approx t$ and we shall reconsider this below. Before we shall do this, we read off the autoresponse function

$$
R(t, u)=u^{-(d+1) / 2} f_{\mathrm{OJK}}(t / u), f_{\mathrm{OJK}}(y)=f_{0} y^{d / 4}(y-1)^{-d / 2}
$$

from which we easily recover, using eq. (4), the values of the exponents $a, a^{\prime}$ and $\lambda_{R}$ in the OJK-approximation (where $z=2$ ) as stated in eq. (5). Eq. (B5) is also recovered in the gaussian theory of phase-ordering [23].

A different conclusion was reached by Berthier et al. [21] albeit starting from the same eq. (B3). They quote $\chi(t, s) \sim s^{-1 / 2} F(t / s)$ for $d>2$ which would mean $a=1 / 2$, provided of course that the naïve scaling law $\chi(t, s)=s^{-a} f_{\chi}(t / s)$ could be used. We must therefore reconsider the singularity in eq. (B4) for $\chi(t, s)$. Following [21, 22], one introduces a cut-off parameter $\Lambda^{2}$ (which should be sent to zero at the end) and writes instead of (B3)

$$
\chi(t, s)=\int_{s}^{t} \mathrm{~d} u \frac{(D t)^{d / 4}}{(D u)^{(d+2) / 4}} \int_{\mathbb{R}^{d}} \mathrm{~d} \boldsymbol{k} e^{-k^{2} D\left(t-u+\Lambda^{2}\right)} .
$$

Performing first the integral over $\boldsymbol{k}$ and changing variables, this becomes, up to a normalization constant

$$
\chi(t, s) \sim t^{(1-d) / 2} \int_{s / t}^{1} \mathrm{~d} v v^{-(d+2) / 4}\left(1-v+\Lambda^{2} / t\right)^{-d / 2}
$$

and we must analyze the contribution of the integrand near $v \approx 1$. We decompose the domain of integration $\int_{s / t}^{1}=\int_{s / t}^{1-\varepsilon}+\int_{1-\varepsilon}^{1}$. In the first term, we let $\Lambda^{2} \rightarrow 0$, and $v \simeq 1$ in the first factor of the second term. Then, for $d>2$

$$
\begin{aligned}
\chi(t, s) \simeq & t^{-(d-1) / 2} \int_{s / t}^{1-\varepsilon} \mathrm{d} v v^{-(d+2) / 4}(1-v)^{-d / 2} \\
& +t^{-(d-1) / 2} \int_{1-\varepsilon}^{1} \mathrm{~d} v\left(1-v+\Lambda^{2} / t\right)^{-d / 2} \\
= & t^{-a} F(s / t)+\mathfrak{c}_{1} t^{-1 / 2}\left(\Lambda^{2}\right)^{1-d / 2}+\mathfrak{c}_{2} t^{-a}\left(\varepsilon+\Lambda^{2} / t\right)^{1-d / 2} \\
= & s^{-1 / 2} \cdot \chi_{\infty}(t / s)^{-1 / 2}+s^{-a} f_{\chi}(t / s)
\end{aligned}
$$

where the value $a=(d-1) / 2$ was used. Here $\mathfrak{c}_{1,2}$ and $\chi_{\infty}$ are constants and $F$ and $f_{\chi}$ are scaling functions. In this way, not only we recover the leading term already found in [21] but we also see that the contribution coming form formally integrating the scaling behaviour of $R(t, s)$ merely gives rise to a sub-leading correction. A similar argument can be applied to the case $d=2$ and produces logarithmic corrections. 
Consequently, the mere observation of a scaling law for $\chi(t, s)$ in the scaling regime $t, s \rightarrow \infty$ with $y=t / s$ fixed is not enough to reliably extract the exponent $a$. Indeed, the term expected from naïve scaling $\chi(t, s) \sim s^{-a}$ merely arises as a short-time correction to the leading long-time behaviour of $\chi(t, s)$. It has already been pointed out in the context of the Ising model quenched to $T<T_{c}$ that a similar dominant term not simply related to the autoresponse exponent $a$ occurs in the ZFC-susceptibility for $d \leq 3$ [55]. The straightforward use of $\chi(t, s)$ may hence lead to erroneous values of the exponent $a$ and it is safer to avoid using $\chi(t, s)$ altogether. 
[1] L.C.E. Struik, Physical ageing in amorphous polymers and other materials, Elsevier (Amsterdam 1978).

[2] A.J. Bray, Adv. Phys. 43, 357 (1994).

[3] M.E. Cates and M.R. Evans (eds) Soft and fragile matter, IOP Press (Bristol 2000).

[4] L.F. Cugliandolo, in Slow Relaxation and non equilibrium dynamics in condensed matter, Les Houches Session 77 July 2002, J-L Barrat, J Dalibard, J Kurchan, M V Feigel'man eds (Springer, 2003); cond-mat/0210312.

[5] C. Godrèche and J.M. Luck, J. Phys. Cond. Matt. 14, 1589 (2002).

[6] A. Crisanti and F. Ritort, J. Phys. A36, R181 (2003)

[7] M. Henkel, Adv. Solid State Phys. 44, 389 (2004).

[8] N. Kawashima and H. Rieger, in Frustrated magnetic systems, H. Diep (ed.) (World Scientific, 2004).

[9] P. Calabrese and A. Gambassi, J. Phys. A at press, cond-mat/0410357.

[10] D.S. Fisher and D.A. Huse, Phys. Rev. B38, 373 (1988).

[11] D.A. Huse, Phys. Rev. B40, 304 (1989).

[12] A. Picone and M. Henkel, J. Phys. A35, 5575 (2002).

[13] M. Henkel and M. Pleimling, Europhys. Lett. 69, 524 (2005).

[14] M. Henkel, Nucl. Phys. B641, 405 (2002).

[15] M. Henkel, M. Pleimling, C. Godrèche and J.-M. Luck, Phys. Rev. Lett. 87, 265701 (2001).

[16] L. Schäfer, J. Phys. A9, 377 (1975).

[17] A. Picone and M. Henkel, Nucl. Phys. B688, 217 (2004).

[18] C. Godrèche and J.M. Luck, J. Phys. A33, 1151 (2000).

[19] E. Lippiello and M. Zanetti, Phys. Rev. E61, 3369 (2000).

[20] M. Henkel and G.M. Schütz, J. Phys. A37, 591 (2004).

[21] L. Berthier, J.L. Barrat and J. Kurchan, Eur. Phys. J. B11, 635 (1999).

[22] G.F. Mazenko, private communication (2004).

[23] G.F. Mazenko, Phys. Rev. E69, 016114 (2004).

[24] M. Henkel and M. Pleimling, Phys. Rev. E68, 065101(R) (2003).

[25] S. Abriet and D. Karevski, Eur. Phys. J. B41, 79 (2004).

[26] M. Henkel, A. Picone and M. Pleimling, Europhys. Lett. 68, 191 (2004).

[27] S. Abriet and D. Karevski, Eur. Phys. J. B37, 47 (2004).

[28] M. Pleimling, Phys. Rev. B70, 104401 (2004).

[29] T. Enss, M. Henkel, A. Picone and U. Schollwöck, J. Phys. A37, 10479 (2004).

[30] M. Pleimling and A. Gambassi, Phys. Rev. B at press (2005); (cond-mat/0501483).

[31] V. Dupuis, F. Bert, J.-P. Bouchaud, J. Hammann, F. Ladieu, D. Parker and E. Vincent, cond-mat/0406672.

[32] P.E. Jönsson, H. Yoshino, P. Nordblad, H. Agura Katori and A. Ito, Phys. Rev. Lett. 88, 257204 (2002).

[33] S. Jiménez, V. Martín-Mayor, G. Parisi and A. Tarancón, J. Phys. A36, 10755 (2003).

[34] G.F. Rodriguez, G.G. Kenning and R. Orbach, Phys. Rev. Lett. 91, 037203 (2003).

[35] M. Picco, F. Ricci-Tersenghi and F. Ritort, Eur. Phys. J. B21, 211 (2001).

[36] A. Maiorano, E. Marinari and F. Ricci-Tersenghi, cond-mat/0409577.

[37] I.S. Suzuki and M. Suzuki, Phys. Rev. B68, 094424 (2003).

[38] M. Henkel, M. Paessens and M. Pleimling, Europhys. Lett. 62, 644 (2003)

[39] W. Zippold, R. Kühn and H. Horner, Eur. Phys. J. B13, 531 (2000).

[40] E.V. Colla, L.K. Chao, M.B. Weissman and D.D. Viehland, Phys. Rev. Lett. 85, 3033 (2000).

[41] M. Rheinstädter, K. Knorr and H. Rieger, Phys. Rev. B69, 144427 (2004).

[42] E.M. Wright, J. London Math. Soc. 10, 286 (1935); Proc. London Math. Soc. 46, 389 (1940); J. London Math. Soc. 27, 256 (1952) erratum.

[43] P.O. Mari and I.A. Campbell, Phys. Rev. B65, 184409 (2002).

[44] H.G. Ballesteros, A. Cruz, L.A. Fernández, V. Martín-Mayor, J. Pech, J.J. Ruiz-Lorenzo, A. 
Tarancón, P. Téllez, C.L. Ullod, and C. Ungil, Phys. Rev. B62, 14237 (2000).

[45] J.-O. Andersson, T. Jonsson and J. Mattsson, Phys. Rev. B54, 9912 (1996).

[46] E.V. Colla, L.K. Chao and M.B. Weissman, Phys. Rev. B63, 134107 (2001).

[47] E. Vincent, V. Dupuis, M. Alba, J. Hammann and J.-P. Bouchaud, Europhys. Lett. 50, 674 (2000).

[48] V. Dupuis, thèse de doctorat (Paris XI Orsay, 2002).

[49] V. Dupuis, E. Vincent, J.-P. Bouchaud, J. Hammann, A. Ito and H.A. Katori, Phys. Rev. B64, 174204 (2001).

[50] R.L. Leheny and S.R. Nagel, Phys. Rev. B57, 5154 (1998).

[51] V. Dupuis, E. Vincent, M. Alba and J. Hammann, Eur. Phys. J. B29, 19 (2002).

[52] I. R. Pimentel, T. Temesvari and C. De Dominicis, Phys. Rev. B65, 224420 (2002);

T. Temesvari, C. De Dominicis and I. R. Pimentel, Eur. Phys. J. B25, 361 (2002).

[53] H.K. Janssen, in G. Györgyi et al. (eds) From Phase transitions to Chaos, World Scientific (Singapour 1992), p. 68

[54] A.J. Bray, ICTP summer school "Statistical physics of frustrated systems" (August 1997), http://www.ictp.trieste.it/ pub_off/sci-abs/smr1003/index.html.

[55] M. Henkel, M. Paessens and M. Pleimling, Phys. Rev. E69, 056109 (2004). 